First publ. in: Journal de Physique / Colloques / Suppléments 43 (1982), C9, pp. 489-492

\title{
PRESSURE DEPENDENCE OF THE LOW TEMPERATURE ACOUSTIC ANOMALIES IN VITREOUS SILICA
}

\author{
U. Barte11 and S. Hunklinger \\ Max-PZanck-Institut für Festkörperforschung, Heisenbergstr. 1, \\ 7000 Stuttgart 80, F.R.G.
}

Résumé. - Nous avons mesuré pour la première fois 1'atténuation ultrasonique dans la silice vitreuse SUPRASIL Wl sous haute pression (jusqu'à 4,2 kbar) à basse température (jusqu'à $60 \mathrm{mK}$ ). A basse température, contrairement à notre attente, on observe seulement une augmentation modérée de $1^{\prime}$ absorption ( $25 \%$ ). A plus haute température, une forte augmentation de 1 'atténuation a été observée.

Abstract. - For the first time we have measured the ultrasonic attenuation in quartz gLasS SUPRASIL W1 under high pressure (up to $4.2 \mathrm{kbar}$ ) down to temperatures of $60 \mathrm{mK}$. At low temperatures $(T<4 K)$, contrary to our expectation, only a moderate increase of absorption $(\sim 25 \%)$ is observed. At higher temperature a strong rise of attenuation has been observed.

1. Introduction. - Anomalous low temperature properties have been found in many disordered solids $/ 1 /$. These anomalies are explained by the tunneling model /2,3/ with the assumption that groups of atoms posess at least two equilibrium positions which are nearly equal in energy. Little is known about the mechanism which couples the tunneling state to an external strain field.

From acoustic measurements the deformation potential is known to be relatively high namely $1 \mathrm{eV}$. Consequently, one expects a strong influence of an applied external pressure.

If the deformation potential would have the same say negative sign for all tunneling states the application of a pressure of $4 \mathrm{kbar}$ should increase the level splitting by about 150K, provided that the linear relationship between pressure and level splitting still holds. Since no tunneling stateshavingan energy splitting less than $150 K$ would exist, the relaxation absorption mainly caused by tunneling states with an energy 3kT should not be observable any more up to a temperature of $50 \mathrm{~K}$. However, it is very likely that the deformation potential can have the opposite sign at different tunneling states. In this case the influence of pressure would be cancelled provided that the density of states is constant and extends to an energy larger than the change achieved by pressure. Therefore, the study of low temperature acoustic absorption under pressure is regarded to be an important means which can provide information about the density of states and the coupling of tunneling states to the lattice.

In tetrahedrically coordinated glass a strong absorption peak is observed at higher temperatures due to thermally activated structural relaxation. In vitreous silica a broad absorption maximum is found around 50K. Several models /6,7,8/ account for this process assuming the motion of an oxygen atom perpendicular or in the direction of the Si-o-bond. Higher pressure could lead either to an increase of the activation energy or to a change of the number of relaxing centers.

2. Experimental technique. - To generate static pressures we utilized a pressure cell similar to that used by Gey et al. Fig. 1 shows a cross section of the pressure cell which was entirely made of CuBe. For acoustic measurements, the sample under pressure is bonded to a window made of a sapphire. On the outer side of the window a quartz transducer is bonded which radiates the ultrasonic waves into the sample. Because of the high attenuation of vitreous silica around 50K, only one reflected pulse from the sample could be observed. Neglecting bond losses, the sample attenuation $\alpha(T)$ may be calculated from the equation

$$
\log A(T)=2 \operatorname{lo}(T)-\log \left(1-R^{2}\right)
$$


Fig. 1: Schematic of the pressure cell

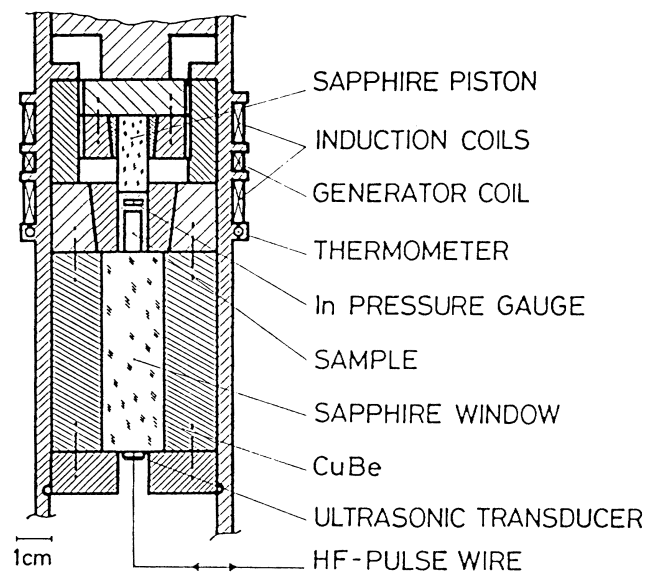

where A ('I') is the amplitude ratio of the reflection from the sample to that from the sapphire-sample interface, $\boldsymbol{l}$ is the length of the sample and $\mathrm{R}$ the stress reflection coefficient at the sapphire-sample boundary. A sapphire slab is bonded to the other end of the sample to enhance the reflection coefficlent.

The pressure was determined at low temperatures by measuring the magnetic susceptibility of an indium probe. To determine the pressure distribution the width of the superconducting transition temperature was monitored. Since the pressure could also be measured at room temperature using the common ruby gauge, the pressure drop could be determined to be roughly 50\% when cooling to liquid Helium.

3. Experimental results. - Fig. 2 shows the acoustic attenuation in SUPRASIL W1 for longitudinal sound waves at $43 \mathrm{MHz}$ for various pressures. At higher temperatures, the most prominent feature at zero pressure is a broad absorption maximum at 50K. When pressure up to $4.2 \mathrm{kbar}$ is exerted on the sample, the peak height increases by a factor of two, while the maximum shifts to 90K. This shift corresponds to an increase of the activation energy from $570 \mathrm{~K} / 5 /$ to $950 \mathrm{~K}$ resulting in a mean pressure derivative of $\partial \mathrm{V} / \mathrm{k} \partial \mathrm{p}=84 \mathrm{~K} / \mathrm{kbar}$.

The attenuation at the Low temperature peak $(\sim 4 \mathrm{~K})$ is slight $\perp$ y increased but no shift with pressure could be detected. Between $0.3 \mathrm{~K}$ and $60 \mathrm{mK}$ the attenuation remains constant. 'l'o extend the temperature range over which the attenuation varies, we also have measured the acoustic attenuation at $200 \mathrm{MHz}$ below $4 \mathrm{~K}$. Fig. 3 shows the ultrasonic attenuation in that temperature region. Although only a pressure of $1.2 \mathrm{kbar}$ has been applied a clear increase of the absorption throughout the temperature range $0.4-4 \mathrm{~K}$ could be observed. The temperature dependence is unchanged but the absorption increases by roughly $25 \%$.

4. Discussion. - The acoustic loss due to a relaxation process is described by the Debye formula. The high temperature peak is ascribed to a thermally activated structural relaxation with a relaxation time given by the Arrhenius formula $\tau=\tau_{0} \exp (\mathrm{V} / \mathrm{kT})$ where $\mathrm{V}$ is the activation energy $/ 5 /$. Since $\mathrm{V}$ is distributed, the Debye formula has to be weighted by the distribution function $P(V)$ and averaged /4/ 


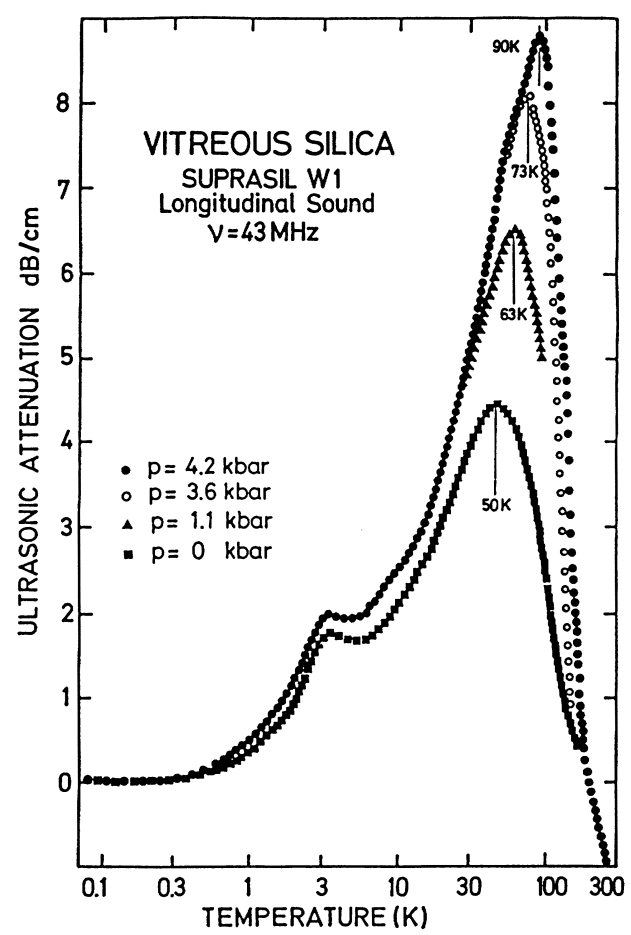

Fig.2: Temperature dependence of the acoustic absorption at various pressures

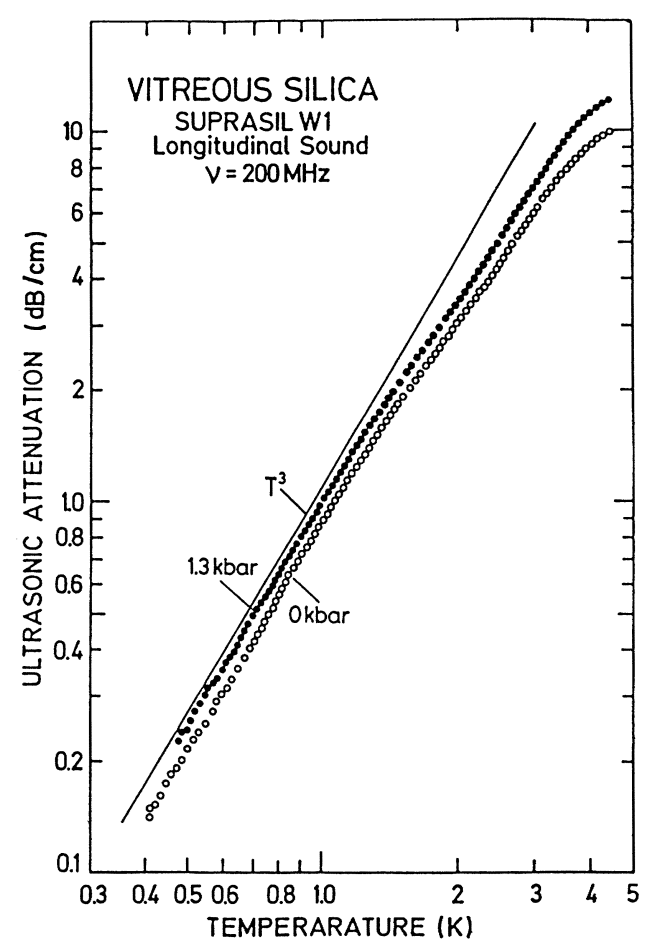

Fig. 3: Temperature dependence of the attenuation at $200 \mathrm{MHz}$ below $4 \mathrm{~K}$.

$$
l_{\text {th.a. }}^{-1}=\frac{D^{2}}{4 \rho v^{2} k T} \int_{0}^{\infty} \operatorname{dVP}(v) \frac{\left.\omega^{2} \tau ! V\right)}{1+\omega^{2} \tau^{2}(V)}
$$

where $\rho, v, D$ are the mass density, sound velocity and the deformation potential, respectively .

In our experiment the peak temperature rises by nearly a factor of two implying that the peak height at $\omega \tau=1$ should decrease by the same amount. Contrary to this prediction the absorption rises, too. Neither $\rho$ nor $v$ can account for this change. A strong variation of the deformation potential $D$ is also very unlikely since this would require a considerable change in the structure of the glass. The only plausible explanation is the increase of the number of relaxing centers by a factor of four.

It is tempting to suggest that with increasing pressure the bond angle of the oxygen defects decreasesresulting in an increase of the activation energy and at the same time creating new relaxation centers.

The acoustic absorption below $10 \mathrm{~K}$ shows less variation with pressure, although at first sight one would expect a much greater effect. The relaxation contribution to the acoustic attenuation by $N$ similar tunneling states, characterized by the tunneling splitting $\Delta_{0}$ and the asymmetry $\Delta$, is given by

$$
\ell_{r e l}^{-1}=\frac{N \bar{D}^{2}}{\rho v^{3}} \frac{\partial f}{\partial E} \frac{\omega \tau^{1}}{1+\omega^{2} \tau_{1}^{2}}
$$


where $\bar{D}=\gamma \Delta / E$ is proportional to the deformation potential $\gamma$, which couples the asymmetry $\Delta$ of the tunneling state to the strain field. $f$ is the population difference in thermal equilıbrıum and $\tau$ the relaxation time. Due to $\partial \mathrm{f} / \partial \mathrm{E}$ in Eq. 3 only tunneling states with an energy of $\mathrm{E} \simeq 3 \mathrm{kT}$ contribute to relaxation absorption. Since the energy variation with static strain $e_{0}$ due to pressure is given by

$$
\mathrm{E}^{2}=\left(\Delta+2 \gamma \mathrm{e}_{0}\right)^{2}+\Delta_{0}{ }^{2}
$$

and the deformation potential $\gamma$ is known to be about $1 \mathrm{eV} / 3 /$ a pressure of $4 \mathrm{kbar}$ would shift the energy of a TLS by 150K, taking the volume compressibility /9/. Provided, all TLS has the same $\gamma$, a gap in the density of states would appear suppressing the relaxation absorption.

On the other hand, if other tunneling states with different sign of their deformation potential $\gamma$ exist which lower the energy when pressure is applied, their states may fill the gap. Then the relaxation absorption change is due to differences in the relative number of tunneling states with opposite sign of deformation potential, being shifted away from or into the energy region around $E=3 \mathrm{kT}$.

In Fig. 3 the acoustic absorption at low temperatures is well described by a $\mathrm{T}^{3}-1 \mathrm{aw}$, which follows from (3) when the direct process is assumed to describe the $\tau-r e l a-$ xation (i.e. $\tau^{1} \sim E^{3}$ ) and $\omega \tau, \gg 1$. After an average has been taken over all $\Delta$ and $E$, the tunneling model gives $/ 1 /$

$$
\ell_{r e l}^{-1} \propto \overline{\mathrm{P}} \gamma^{2} \mathrm{~T}^{3}
$$

where $\overline{\mathrm{P}}$ is the mean density of states. Again, $\rho$ and $\mathrm{v}$ do not account for the observed change of about 25\%. Since $\gamma \sim \overline{\mathrm{D}}$, similar to the reason given above, only a change in $\overline{\mathrm{P}}$ could contribute to the pressure change.

Summarizing, it may be concluded, that the observed small change of relaxation by tunneling states indicates that there are almost equal numbers of states with positive and negative $\gamma$ and the density of states increases by $\sim 25 \%$ at $3 \mathrm{~K}$ when pressure is applied.

\section{$\underline{\text { References }}$}

/1/ S. Hunklinger and M.v. Schickfus: In "Amorphous Solids", ed. by W.A. Phillips (Springer, Heidelberg 1981), pp 81 - 105

/2/ P.W. Anderson, B.I. Halperin, C. Varma: Phil. Mag. 25, 1 - 9 (1972)

/3/ W.A. Phillips:J. Low Temp. Phys. I, 351 - 360 (1972)

/4/ J. Jäckle, L. Piché, W. Arnold, and S. Hunklinger: J. Non-Cryst. Solids, 20, 365 (1976)

15/ S. Hunklinger and W. Arnold: In "Physical Acoustics", ed. by W.P. Mason, R.N. Thurston (Academic, New York 1976), Vol. XII, pp 155 - 215

/6/ O.L. Anderson, H.E. Bömmel: J. Am. Ceram. Soc. 38, 125 (1955)

/7/ R.E. Strakna: Phys. Rev. 123, 2020 (1961)

/8/ M.R. Vukcevich: J. Non-Cryst. Solids 11, 25 (1972)

19/ O.L. Anderson: In "Progress in very high pressure research", p 225, Wiley N.Y. (1961). 